Wilfrid Laurier University

Scholars Commons @ Laurier

Physics and Computer Science Faculty

Publications

Physics and Computer Science

$9-1978$

\title{
Surface Roughness Measurement Using Dichromatic Speckle Pattern: An Experimental Study
}

Hitoshi Fujii

Virginia Polytechnic Institute and State University

John W.Y. Lit

Wilfrid Laurier University, jlit@wlu.ca

Follow this and additional works at: https://scholars.wlu.ca/phys_faculty

\section{Recommended Citation}

Fujii, Hitoshi and Lit, John W.Y., "Surface Roughness Measurement Using Dichromatic Speckle Pattern: An Experimental Study" (1978). Physics and Computer Science Faculty Publications. 21.

https://scholars.wlu.ca/phys_faculty/21

This Article is brought to you for free and open access by the Physics and Computer Science at Scholars Commons @ Laurier. It has been accepted for inclusion in Physics and Computer Science Faculty Publications by an authorized administrator of Scholars Commons @ Laurier. For more information, please contact scholarscommons@wlu.ca. 


\title{
Surface roughness measurement using dichromatic speckle pattern: an experimental study
}

\author{
Hitoshi Fujii and John W. Y. Lit
}

\begin{abstract}
Surface roughness is studied experimentally by making use of the statistical properties of dichromatic speckle patterns. The rms intensity difference between two speckle patterns produced by two argon laser lines are analyzed in the far field as functions of the object surface roughness and the difference in the two wavenumbers of the illuminating light. By applying previously derived formulas, the rms surface roughness is obtained from rms intensity differences. Glass and metal rough surfaces are used. Other than the scattering arrangement, the experimental setup has a simple spectrometric system and an electronic analyzing circuit.
\end{abstract}

\section{Introduction}

In practice, surface roughness is normally measured by using stylus instruments. One such instrument measures the CLA (center line average height) roughness from $0.05 \mu \mathrm{m}$ to $10 \mu \mathrm{m}$ with a fine diamond point which exerts a small pressure of only $0.1 \mathrm{mg}$. It is, however, a laboratory technique that requires the test object to be physically removed from the process which it serves. The principal drawbacks of this method are its slowness to affect measurements and its effect on the surface being measured. Even with the lowest pressure possible on the stylus, scratches are often left on an aluminum mirror surface under test. To overcome these drawbacks, much work has been carried out studying alternative optical techniques providing a nonmechanical-contact method to measure surface roughness. ${ }^{1}$

Based on theoretical studies of electromagnetic-wave scattering, angular distributions of average intensities scattered from various types of surfaces have been studied extensively by many workers. ${ }^{2,3}$ One of the simplest methods can determine the rms surface roughness by measuring the mean intensity of light scattered into the specular direction. However, since the accuracy of this method depends on the surface

When this work was done both authors were with Université Laval, Laboratoire de Recherches en Optique et Laser, Quebec, P.Q. H. Fujii is now with Virginia Polytechnic Institute \& State University, Blacksburg, Virginia 24061; and J. W. Y. Lit is with Wilfrid Laurier University, Physics Department, Waterloo, Ontario N2L 3C5.

Received 27 February 1978.

0003-6935/78/0901-2690\$0.50/0.

(C) 1978 Optical Society of America. profile, it may not be used fully in practical measurements. ${ }^{1,4}$ Therefore, other optical methods have been more commonly used. By observing the deformation of fringe patterns in an interferogram or that of the shadow of a narrow slit imaged onto a rough surface, the surface profile, maximum height, and rms roughness can be estimated. ${ }^{1}$

Recently, various speckle techniques have been studied to measure surface roughness properties. ${ }^{5}$ In particular, the correlation function of speckle patterns produced at different wavelengths has been studied under various conditions of surface structure, illumination, and system observation. ${ }^{5-12}$ It has been pointed out that the two-wavelength speckle patterns decorrelate with an increase of object surface roughness or with an increase in difference in the two wavenumbers. The spatial coherence function of the speckling field has also been applied to determine the correlation function of surface height variations. ${ }^{13}$ Some holographic or speckle interferometric methods have been reported and improved to give real time measurements of surface roughness. $5,14,15$

Considerable effort has also been made in investigating the relationship between object surface roughness and speckle pattern contrast.,5,16-23 In particular, by measuring the contrast variation in monochromatic speckle patterns, ${ }^{16-19}$ a very useful technique has been proposed and studied to determine rms surface roughness, waviness, and the correlation length of surface height variations. ${ }^{20-23}$ Since the contrast can be calculated systematically for various types of surfaces, the method seems to be more practical for in-process measurements. However, with a $\mathrm{He}-\mathrm{Ne}$ laser, the effective measuring range of optical roughness of that method is limited to $0-0.2 \mu \mathrm{m}$; this is relatively narrower than the current stylus method. 


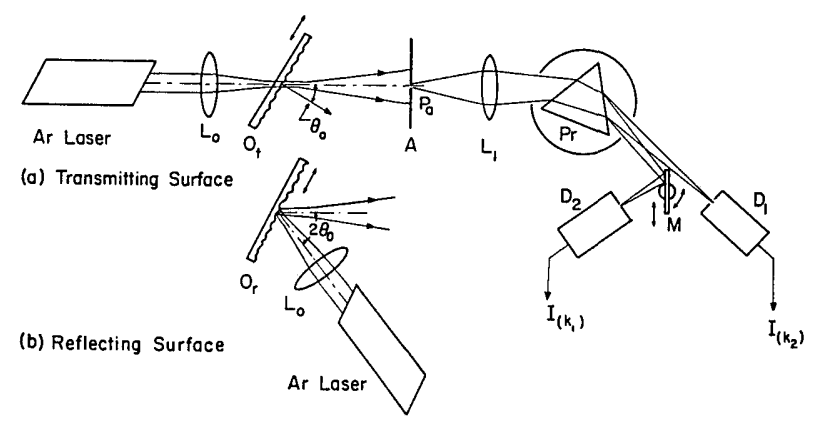

Fig. 1. Geometry of the optical system for (a) the transmitting surface and (b) the reflecting surface. The polichromatic speckle pattern in the observing plane $A$ is analyzed by a simple spectrometer.

In order to extend the measuring range of $\mathrm{rms}$ roughness up to a few microns, we have studied theoretically the differences in two speckle patterns produced by dichromatic illumination. ${ }^{24}$ A new method has been proposed to determine the rms roughness by measuring the rms difference in two normalized intensity distributions in dichromatic speckle patterns. In this paper, we report on an experimental study of the proposed method. Various grades of ground glass plates and metal surfaces are taken as test surfaces. The experimental results are compared with the theoretical curves and with those of a similar method proposed recently by Wykes. ${ }^{25}$

\section{Experimental Arrangement}

Figure 1 shows the schematic diagram of the experiment. An argon laser in multiline operation is used as a polichromatic source. The laser beam is focused by a converging lens $L_{0}$ onto an object's rough surface; this is shown as $O_{t}$ [Fig. 1(a)] in the case of a transmitting surface and as $O_{r}$ [Fig. 1(b)] in the case of a reflecting surface. The incident angle is $\theta_{0}$. The light scattered from the object surface produces a polichromatic speckle pattern in the far-field plane $A$. The distance from the object surface to the observing plane $A$ is 56 $\mathrm{cm}$. The diameter of the laser spot on the object surface is $0.32 \mathrm{~mm}$, which is much larger than the correlation length of the surface height variation of ordinary rough surfaces. The average speckle size on the plane $A$ is calculated at $1.1 \mathrm{~mm} .{ }^{26}$ A small pinhole $P_{a}$ is placed at the on-axis point of the plane $A$, detecting the polichromatic speckle intensity. The diameter of the pinhole $P_{a}$ is $0.1 \mathrm{~mm}$, which is much smaller than the average size of the speckles. The system behind the pinhole $P_{a}$ is a simple spectrometer consisting of a lens $L_{1}$, a prism $P_{r}$, a small mirror $M$, and two photomultipliers $D_{1}$ and $D_{2}$. Two particular wavelengths are selected by changing the position of the mirror $M$ and the detector $D_{1}$. By translating the object surface $O_{t}$ or $O_{r}$ across the illuminating light with a constant velocity, the polichromatic speckle pattern at plane $A$ and accordingly the two intensities at detectors $D_{1}$ and $D_{2}$ vary with time. The two intensity variations of the dichromatic speckle pattern are converted to signal currents, and their differences are studied as functions of the object surface roughness and the wavenumbers of the illuminating light. ${ }^{24}$ Since the argon laser is polarized linearly and the plane of polarization is set perpendicular to the plane of incidence (purely horizontal polarization), the scattered light from the rough surface is not depolarized at the detecting point $P_{a}$ in the plane of incidence. ${ }^{2}$ The previous theoretical study based on the scalar diffraction theory is therefore applicable to this experimental study.

In the theory, ${ }^{24}$ we have introduced the rms difference $\tilde{V}$ and found a useful equation,

$$
\begin{gathered}
\tilde{V}\left(\sigma_{s} ; k_{1}, k_{2}\right)=\left\langle\left[\frac{I\left(k_{1}\right)}{\left\langle I\left(k_{1}\right)\right\rangle}-\frac{I\left(k_{2}\right)}{\left\langle I\left(k_{2}\right)\right\rangle}\right]^{2}\right\rangle^{1 / 2} \\
=\left[\frac{\left\langle I\left(k_{1}\right)^{2}\right\rangle}{\left\langle I\left(k_{1}\right)\right\rangle^{2}}+\frac{\left\langle I\left(k_{2}\right)^{2}\right\rangle}{\left\langle I\left(k_{2}\right)\right\rangle^{2}}-\frac{2\left\langle I\left(k_{1}\right) I\left(k_{2}\right)\right\rangle}{\left\langle I\left(k_{1}\right)\right\rangle\left\langle I\left(k_{2}\right)\right\rangle}\right]^{1 / 2} \\
=\left(2\left\{1-\exp \left[-\sigma_{s}^{2}\left(k_{1}-k_{2}\right)^{2}\right]\right\}\right)^{1 / 2}
\end{gathered}
$$

where $I\left(k_{1}\right)$ and $I\left(k_{2}\right)$ are two intensity variations of the dichromatic speckle pattern, $k$ is the wavenumber (= $2 \pi / \lambda)$, and $\sigma_{s}$ is the rms optical roughness, that is, the rms optical path fluctuation due to the object surface roughness.

Figure 2 shows some of the theoretical curves of the rms difference $\tilde{V}\left(\sigma_{s} ; k_{1}, k_{2}\right)$ plotted against the optical roughness $\sigma_{s}$. The curves are evaluated for the cases given by pairs of lines of an argon laser. The sensitivity can be altered by changing the wavelength pair. In this experiment, we chose two pairs: (a) $5145-4765 \AA$ and (b) 5145-4965 A. In Eq. (1), both signals $I\left(k_{1}\right)$ and $I\left(k_{2}\right)$ are divided by their mean values which are unknown until the end of each scan. Therefore, the signals must be stored temporally in a data recording system and played back to a calculating system of the rms difference $\tilde{V}$ with their measured mean values. With Eq. (2), since the five different terms $\left\langle I\left(k_{1}\right)\right\rangle,\left\langle I\left(k_{2}\right)\right\rangle,\left\langle I\left(k_{1}\right)^{2}\right\rangle$, $\left\langle I\left(k_{2}\right)^{2}\right\rangle$, and $\left\langle I\left(k_{1}\right) I\left(k_{2}\right)\right\rangle$ can be calculated simultaneously, the rms difference is evaluated immediately after each scan.

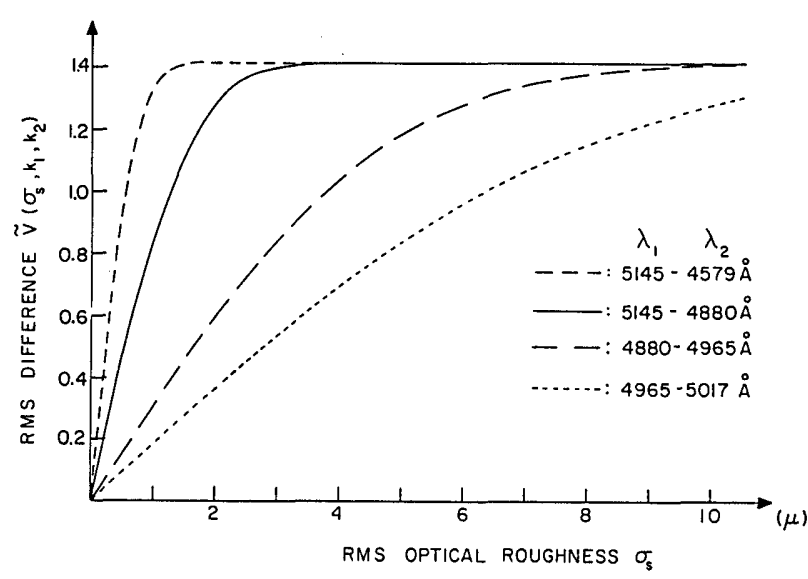

Fig. 2. Theoretical curves of the rms difference $\tilde{V}\left(\sigma_{s} ; k_{1}, k_{2}\right)$. The curves are calculated for cases of dichromatic illumination using pairs of lines of an $A_{r}$ laser. 
In this experiment, we have set up a simple analyzer system to calculate the five mean values and substitute them into Eq. (2) to evaluate the rms difference $\tilde{V}$. Figure 3 shows the block diagram of the system. Each of the two signals from the photomultipliers passes through a preamplifier $A_{1}$ or $A_{2}$ and an integrater $G_{1}$ or $G_{2}$ to yield its mean value $\left\langle I\left(k_{1}\right)\right\rangle$ or $\left\langle I\left(k_{2}\right)\right\rangle$. Two signals are also fed into an analog multiplier $M$ and an integrater $G_{3}$. By changing the selector switch, we have the values $\left\langle I\left(k_{1}\right)^{2}\right\rangle,\left\langle I\left(k_{2}\right)^{2}\right\rangle$, and $\left\langle I\left(k_{1}\right) I\left(k_{2}\right)\right\rangle$. The time of integration is $10 \mathrm{sec}$, and during this period 8.4 $\mathrm{mm}$ of the object rough surface is scanned. Eight different grades of ground glass plates $(600 \sim 80)$ serve as transmitting test surfaces. For comparison, their roughnesses are measured beforehand mechanically by the stylus instrument Talysurf-4. The center-lineaverage roughness CLA of those glass plates is from 0.34 $\mu \mathrm{m}$ to $5.1 \mu \mathrm{m}$ (cut off, $0.8 \mathrm{~mm}$ ). On the other hand, four metal surfaces of a roughness standard (Rugotest 104) processed by surface grinding are chosen to serve as reflecting surfaces. Their CLA roughness has been given as $0.1-0.8 \mu \mathrm{m}$. Since the profile of these two kinds of surfaces is approximately expressed by Gaussian random functions, the rms roughness $R_{s}$ is obtained from CLA by using

$$
R_{s}=(\pi / 2)^{1 / 2} \cdot \text { CLA. }
$$

For the transmitting surfaces, the optical roughness $\sigma_{s}$ is given by

$$
\begin{aligned}
\sigma_{s} & =R_{s}\left(n_{1} \cos \theta_{1}-\cos \theta_{0}\right), \\
n_{1} \sin \theta_{1} & =\sin \theta_{0} \quad \text { (Snell's refraction law), }
\end{aligned}
$$

where $n_{1}$ is the refractive index of the glass plate, $\theta_{0}$ is the incident angle of laser light, and $\theta_{1}$ is the refracting angle in the glass plate. For reflecting surfaces, $\sigma_{s}$ is related to $R_{s}$,

$$
\sigma_{s}=2 R_{s} \cos \theta_{0} .
$$

The effects of incident angle on the rms difference are studied in Sec. III.B.

\section{Results and Discussion}

\section{A. Measurement of Root Mean Square Roughness}

Figure 4 compares the experimental data with theoretical results. The experimental data of the rms difference $\tilde{V}$ are plotted against the optical roughness $\sigma_{s}$ converted from the values of roughness measured by a mechanical stylus. The measurements of the rms difference were repeated ten times for each surface under two different conditions of dichromatic illumination. In this case, the incident light is normal to the surfaces. The solid line is the theoretical curve for a green and violet light pair (5145-4765 $\AA$ ), and the broken line is that for a green and blue light pair (5145-4965 $\AA$ ). It is found that the experimental results are in fairly good agreement with the theoretical curves in both cases. The upper curve is fairly linear from $0 \mu \mathrm{m}$ to $1 \mu \mathrm{m}$, while

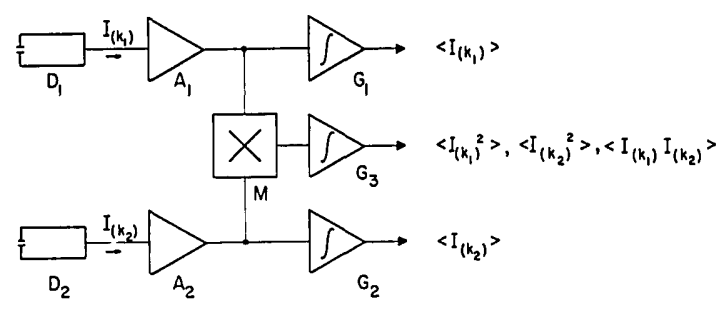

Fig. 3. Block diagram of the analyzing circuit. $D_{1}, D_{2}$ : photomultipliers; $A_{1}, A_{2}$ : preamplifiers; $M$ : analog multiplier; $G_{1}, G_{2}, G_{3}$ : integrators.

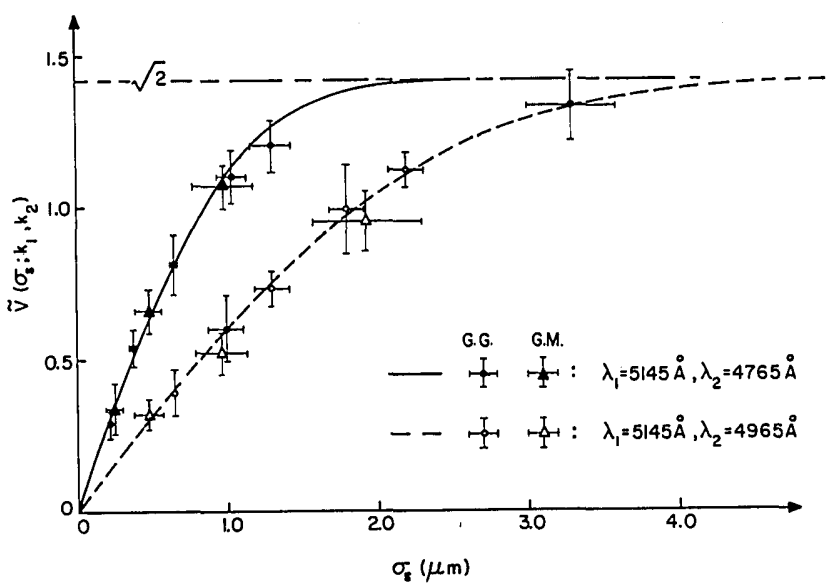

Fig. 4. Experimental results of the rms difference $\tilde{V}\left(\sigma_{s} ; k_{1}, k_{2}\right)$ compared with theoretical curves. The data are plotted by using the values of roughness measured by a mechanical stylus: The circles are for ground glass surfaces (G.G.), and the triangles are for ground metal surfaces (G.M.). The solid symbols are for the wavelength pair $\lambda=5145 \AA, 4765 \AA$. The blank symbols are for the wavelength pair $\lambda=5145 \AA, 4965 \AA$. The bars indicate standard deviations.

the lower curve is so from $0 \mu \mathrm{m}$ to $2 \mu \mathrm{m}$. Therefore, it is seen that the effective measuring range is actually changed from $0-1 \mu \mathrm{m}$ to $0-2 \mu \mathrm{m}$ in the rms optical roughness by changing the light pair. Comparing this with the results obtained by Wykes, ${ }^{25}$ we have a much higher accuracy, partly because we have obtained more sample points to reduce the error. From Eq. (3), we derive

$$
\sigma_{s}=\left[-\ln \left(\tilde{V}^{2} / 2\right)\right]^{1 / 2} /\left|k_{1}-k_{2}\right|,
$$

which enables us to calculate the rms optical roughness $\sigma_{s}$ directly by substituting the predetermined value of $k_{1}-k_{2}$ and the value of the rms difference $\tilde{V}$ measured experimentally. Hence, with the use of Eqs. (5)-(7), the actual rms roughness $R_{s}$ of a surface can be determined by the present speckle method.

In order to show the correlation between the measured values of the rms roughness obtained with the mechanical stylus and those given by the speckle method, the experimental results in Fig. 4 are tran- 


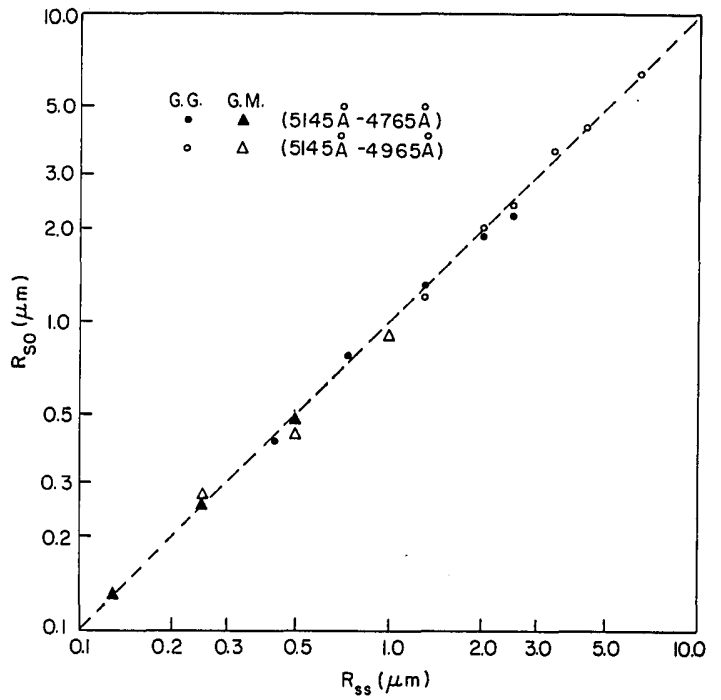

Fig. 5. The correlation between the two experimental values of $\mathrm{rms}$ roughness. $R_{s s}$ is the rms surface roughness measured by a mechanical stylus method. $R_{s o}$ is that by the present speckle method.

scripted into Fig. 5. $R_{s s}$ is the rms roughness obtained by the mechanical stylus method. $R_{s o}$ is that obtained by the speckle method. The correlation between the two sets of data is more than $90 \%$ from $0.13 \mu \mathrm{m}$ of a metal surface to $6 \mu \mathrm{m}$ of a ground glass plate.

\section{B. Effects of Incident Angle}

Equations (5) and (7) indicate that the optical surface roughness is a function of incident angle $\theta_{0}$ of the laser beam. It is expected, therefore, that the measuring range or the sensitivity can be altered by varying the incident angle. In Figs. 6(a) and 6(b), some of the experimental results of the rms difference $\tilde{V}$ are plotted against the incident angle $\theta_{0}$ for both type of surfaces. The solid lines show the theoretical curves calculated by using Eqs. (3) and (5)-(7). It is seen from Fig. 6(a) that, for the transmitting surfaces, the rms difference increases with the incident angle increase. Therefore, it is possible to increase the sensitivity to some extent by increasing the incident angle.

On the contrary, Fig. 6(b) indicates that in the case of reflecting surfaces, the rms difference decreases with incident angle increase. This seems to be reasonable from the well known fact that the effective optical roughness of reflecting surfaces decreases with oblique incidence. Hence, the sensitivity can be reduced by as much as a factor of 0.5 by increasing the incident angle.

\section{Laser-Spot Size}

Actually, the amplitude distributions in the neighborhood of the focal point or beam waist are different for each component of the dichromatic light. This means that the size of the laser-beam spot in the rough surface from which the light is scattered and superposed at the observing point is actually different for the two chosen wavelengths. Moreover, in the theoretical study, ${ }^{24}$ it has been assumed that the rough surface is illuminated uniformly within an aperture, while in the present experiment, the amplitude distributions have Gaussian forms. Nevertheless, no significant error due to these different conditions has been found between the theoretical and the experimental results. This fact means that the rms difference $\tilde{V}$ is actually quite insensitive to the shape and the size of the laser spot in the scattering surface, if the laser spot illuminates many scattering elements. ${ }^{27}$

It is known that with an increase of roughness, the correlation length of the surface height increases. Then the principal scattering elements become considerably larger in the illuminated area. Since the central limiting theory is no longer valid for this condition, the contrast in the resulting speckle pattern is enhanced due to non-Gaussian statistics. ${ }^{11}$ In our experiment, this fact has been observed with a very bright spot of all wavelength components outstanding in the homogeneous polichromatic speckle pattern. This condition is not acceptable for the present speckle method based on the Gaussian statistics. In order to eliminate the speckle enhancement, the spot size is chosen as large as possible within the limit of the resolving power of the detecting pinhole $P_{a}$ (Fig. 1). For very rough surfaces, however, due to the enlarged spot size, the light energy is scattered so widely that the intensity is no longer an efficient parameter for detection. Therefore, the practical measuring range may be limited by that efficiency. Within the measuring range of the present experiment, all test surfaces have produced nonenhanced speckle patterns.

Another interest in the size of the laser spot is its relation to the so-called roughness width cutoff defined empirically in current stylus methods. The standard cutoff value for the measurements of CLA roughness is chosen to be $0.8 \mathrm{~mm}$ for normal surfaces, or $0.25 \mathrm{~mm}$ for

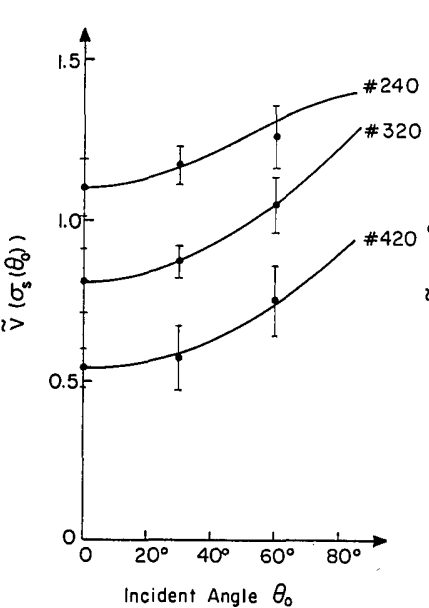

(a)

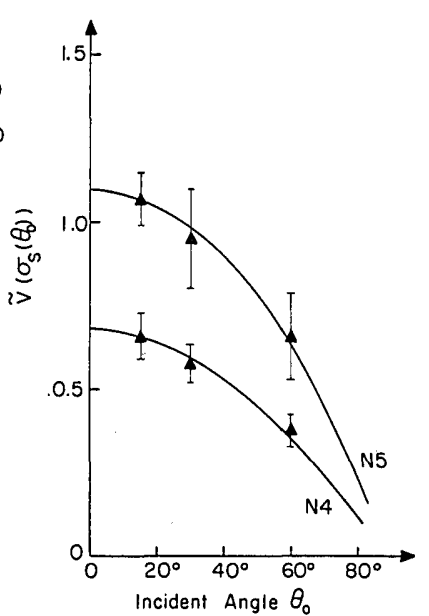

(b)
Fig. 6. Effect of the incident angle $\theta_{0}$ of the laser beam on the rms difference $\tilde{V}$. (a) Transmitting ground glass surfaces. (b) Reflecting ground metal surfaces: $N 4(\mathrm{CLA}=0.2 \mu \mathrm{m})$ and $N 5(=0.4 \mu \mathrm{m})$. The wavelength pair is $\lambda=5145 \AA, 4765 \AA$. 
very smooth surfaces. The size of the laser spot in this experiment is $0.32 \mathrm{~mm}$, which is regarded as the maximum wavelength of the roughness contributing to the production of speckle patterns. Since the size is of the order of the standard cutoff values of the stylus method, both experimental results in Fig. 5 are in good agreement.

\section{Conclusion}

It now becomes possible to estimate rms surface roughness by measuring the rms difference in two normalized intensity variations in a dichromatic speckle pattern produced in the far field of the object surface. The effective measuring range in this experimental study is $0.4-6 \mu \mathrm{m}$ for the ground glass surfaces and $0.1-1$ $\mu \mathrm{m}$ for the ground metal surfaces. The method is available for random rough surfaces processed by such methods as grinding, lapping, and polishing, which produce polichromatic speckle patterns. The measuring range of the present method actually covers one of the important regions of roughness ordinarily encountered in those processes. For surfaces with periodic profiles, our method may not be applied, because these surfaces act as a phase grating for the polichromatic illumination with resulting diffraction patterns having periodic forms instead of random forms.

This work is supported by the National Research Council of Canada. We thank G. Tremblay in the Department of Mechanical Engineering, Laval University, for lending us a few roughness standards and for helping us measure the surface roughness of ground glass plates with Talysurf-4.

\section{References}

1. J. M. Bennett, Appl. Opt. 15, 2705 (1976).

2. P. Beckmann and A. Spizzichino, The Scattering of Electromagnetic Waves from Rough Surfaces (Pergamon, Oxford, 1963).

3. E. P. Lavin, Specular Reflection; Monographs on Applied Optics 2 (American Elsevier, New York, 1971); J. C. Richmond and J. J. Hsia, J. Res. Nat. Bur. Stand. Sect. A: 80, 207 (1976).

4. R. A. Sprague, Appl. Opt. 11, 2811 (1972).

5. Special issue on speckle phenomena, J. Opt. Soc. Am. 66, No. 11 (1976).

6. J. W. Goodman, Stanford Electronics Laboratories; TR2303-1 (SEL-63-140) (1963).

7. N. George and A. Jain, Appl. Phys. 4, 201 (1974).

8. G. Tribillon, Opt. Commun. 11, 172 (1974).

9. G. Parry, Opt. Commun. 12, 75 (1974).

10. H. M. Pedersen, Opt. Acta 22, 15, 523 (1975).

11. E. Jakeman and P. N. Pusey, J. Phys. A: Math. Nucl. Gen. 8, 369 (1975).

12. N. George, A. C. Livanos, J. A. Roth, and C. H. Papas, Opt. Acta 23, 367 (1976).

13. K. Nagata and T. Umehara, Jpn. J. Appl. Phys. 12, 694 (1973).

14. W. B. Ribbens, Appl. Opt. 11, 807 (1972).

15. D. Léger, E. Mathieu, and J. C. Perrin, Appl. Opt. 14, 872 (1975).

16. H. Fujii and T. Asakura, Opt. Commun. 11, 35 (1974).

17. H. M. Pedersen, Opt. Commun: 12, 156 (1974).

18. H. M. Pedersen, in Ref. 5, p. 1204.

19. N. George, A. Jain, and R. D. S. Melville, Appl. Phys. 7, 157 (1975).

20. H. Fujii, T. Asakura, and Y. Shindo, in Ref. 5, p. 1217.

21. H. Fujii and T. Asakura, Appl. Opt. 16, 180 (1977).

22. H. Fujii and T. Asakura, J. Opt. Soc. Am. 67, 1171 (1977).

23. J. Uozumi and T. Asakura, Opt. Laser Technol. 9, 177 (1977).

24. H. Fujii and John W. Y. Lit, Opt. Commun. 22, 231 (1977).

25. C. Wykes, Opt. Acta 24, 517 (1977).

26. J. C. Dainty, Ed., Laser Speckle and Related Phenomena (Springer, Berlin, 1975).

27. H. Fujii and T. Asakura, Opt. Commun. 21, 80 (1977). 